\title{
Unitary quantum gates, perfect entanglers and unistochastic maps
}

\author{
Marcin Musz ${ }^{1}$, Marek Kuś ${ }^{1}$, and Karol Życzkowski ${ }^{1,2}$ \\ ${ }^{1}$ Center for Theoretical Physics, Polish Academy of Sciences, Al. Lotników 32/44, 02-668 Warszawa, Poland \\ ${ }^{2}$ Institute of Physics, Jagiellonian University, ul. Reymonta 4, 30-059 Kraków, Poland
}

\begin{abstract}
Non-local properties of ensembles of quantum gates induced by the Haar measure on the unitary group are investigated. We analyze the entropy of entanglement of a unitary matrix $U$ equal to the Shannon entropy of the vector of singular values of the reshuffled matrix. Averaging the entropy over the Haar measure on $U\left(N^{2}\right)$ we find its asymptotic behaviour. For two-qubit quantum gates we derive the induced probability distribution of the interaction content and show that the relative volume of the set of perfect entanglers reads $8 / 3 \pi \approx 0.85$. We establish explicit conditions under which a given one-qubit bistochastic map is unistochastic, so it can be obtained by partial trace over a one-qubit environment initially prepared in the maximally mixed state.
\end{abstract}

PACS numbers: 03.65.Ta

Keywords:

\section{INTRODUCTION}

Unitary quantum gates form key ingredients of any quantum algorithm, so they are widely used in the theory of quantum information [1]. A unitary gate acting on a bipartite system $A \otimes B$ is called local, if the unitary matrix has a form of the tensor product, $U=U_{A} \otimes U_{B}$. To process quantum information between both subsystems one needs to use non-local gates, which are not of the product form.

Classification of unitary quantum gates is a subject of a considerable interest [2 [5]. Quantification of the nonlocal properties of unitary gates has been initiated by Zanardi and co-workers [6 8], while several other measures of non-locality were introduced and analyzed in a seminal paper of Nielsen et al. 9]. Since local unitary gates cannot produce quantum entanglement, the nonlocal properties of a given gate $U$ may be characterized by the average (or maximal) degree of entanglement of a transformed separable state $\left|\psi^{\prime}\right\rangle=U\left|\psi_{\text {sep }}\right\rangle$. For instance, the average linear entropy of a random product state transformed by a bi-partite unitary gate leads to its entangling power, introduced by Zanardi et al. [6], and later investigated for various models in $10-16$. An alternative approach to the problem of non-locality based on the mimimal Frobenius distance of an analyzed global unitary matrix to the closest local gate was recently discussed in [17], where a relation to matrix product operator formalism was established.

A given unitary gate $U$ is called a perfect entangler, if there exists a separable state transformed by $U$ into a maximally entangled state [18]. Another class of maximally entangling unitary gates was characterized in [19, 20].

*Electronic address: marek.kus@cft.edu.pl, karol@cft.edu.pl
Two unitary gates are called locally equivalent if they coincide up to local transformations. The general problem of finding necessary and sufficient conditions for local equivalence remains open. The full answer is known in the simplest case of a two-qubit system, since a canonical form of a unitary gate of size four is established and any gate can be uniquely described by a three-components vector called information content [2, 21 23].

In this work we analyze properties of a 'typical' quantum gate. In other words, we are going to average quantities characterizing each gate over a unique, unitarily invariant, Haar measure on the space of unitary matrices. An ensemble generated according to this measure is often referred to as circular unitary ensemble (CUE), the spectrum belongs to the unit circle and the ensemble is invariant with respect to unitary transformation. An exemplary algorithm of generating unitary matrices from this ensemble was discussed in [24]. To study the set of quantum gates we found it useful to define a special circular unitary ensemble (SCUE) containing special matrices with determinant equal to unity.

One of the main result of this paper consists in deriving the probability distribution $P(\vec{a})$ for the information content of a random two-qubit gate, induced by the Haar measure on the unitary group. As an application of this result we compute the relative volume of the set of perfect entanglers with respect to this natural measure. Basing on numerical results performed for unitary gates of larger dimensionalities we are in position to predict asymptotic behavior of the average entanglement entropy of a random unitary gate.

Furthermore, we analyze the class of unistochastic operations, introduced in 25], which can be described by a coupling with an $M$-dimensional environment initially in the maximally mixed state,

$$
\rho^{\prime}=\Phi_{U}(\rho)=\operatorname{Tr}_{\mathrm{env}}\left[U\left(\rho \otimes \frac{\mathbb{1}}{N}\right) U^{\dagger}\right]
$$


The partial trace is performed over the environment described in the Hilbert space $\mathcal{H}_{M}$. If the dimension $N$ of the principal system and the dimension $M$ of the ancillary system are equal, the map is called unistochastic, while it is called $k$-unistochastic if $M=k N$. Thus a unistochastic map $\Psi_{U}$ is determined by a unitary matrix $U$ of size $N^{2}$, while any $k$-unistochastic map is given by a matrix of size $N^{k+1}$.

By construction any unistochastic map is bistochastic, since the maximally mixed state is preserved, $\Psi_{U}(\mathbb{1} / N)=\operatorname{Tr}_{E}\left(\mathbb{1}_{N^{2}}\right) / N^{2}=\mathbb{1}_{N} / N$. On the other hand, the converse is not true, and in this paper we determine sufficient and necessary condition for a one-qubit bistochastic map to be unistochastic.

The name of this class of maps is related to the classical case, in which probability vectors are transformed by stochastic matrices. The matrix is called bistochastic (or doubly stochastic), if it preserves the uniform (maximally mixed) probability vector. A bistochastic matrix $B$ is called unistochastic if there exists an unitary $V$ of the same size such that $B_{i j}=\left|V_{i j}\right|^{2}$. For $N=2$ all bistochastic matrices are unistochastic, but this is not the case already for $N=3$. For higher dimensions the problem finding necessary and sufficient conditions for unistochasticity remains open [26].

In analogy to the classical case, any quantum map determined as in (1) by an orthogonal matrix will be called orthostochastic. In 25] it was shown that for any unistochastic map the spectrum of the corresponding dynamical matrix is given by the Schmidt coefficients of the unitary matrix $U$ treated as an element of the composite Hilbert-Schmidt space. This implies that the entropy of such an operation $S\left(\Psi_{U}\right)$ is equal to the entanglement entropy of the unitary matrix. In other words, a link between non-local properties of a unitary gate acting on a bipartite system and the decoherence induced by an associated unistochastic map acting on a single system can be established.

It is appropriate to mention that the class of quantum maps for which the system is coupled with the environment in the maximally mixed state was already investigated in the literature. Such maps were discussed in the context of quantum information processing [27, 28], and, under the name 'noisy maps', while studying reversible transformations from pure to mixed states [29]. Moreover, Haagerup and Musat [30] analyzed properties of a related class of factorizable quantum maps introduced in [31]. In fact $k$-unistochastic operations coincide with a subset of these quantum maps called exactly factorizable. In general this set is not convex, and its convex hull defines a larger set of maps called strongly factorizable.

Note that a given unitary matrix $U$ of a composite dimension $d=N^{2}$ may play very different roles in the theory of quantum information. Let us specify here three most natural applications:

a) $U \in U\left(N^{2}\right)$ describes a quantum gate acting on a $N \times N$ bi-partite system, and its operator Schmidt decomposition characterizes the non-local properties [3,
[4, 9, 21],

b) $U \in U\left(N^{2}\right)$ determines by Eq. (11) a unistochastic quantum operation, $\rho^{\prime}=\Phi_{U}(\rho)$, acting on an $N$-level system [25],

c) $U \in U\left(N^{2}\right)$ defines a maximally entangled state of a composite, $N^{2} \times N^{2}$ system [32, 33], as $|\psi\rangle=(U \otimes \mathbb{I})\left|\psi_{+}\right\rangle$ where $\left|\psi_{+}\right\rangle=\frac{1}{N} \sum_{j=1}^{N^{2}}|j, j\rangle$.

The paper is organized as follows. Unistochastic operations are analyzed in section II. As any one-qubit unistochastic map is determined by a unitary matrix of order four, we analyze in section III the set of all two-qubit unitary gates. This allows us to characterize the set $\mathcal{U}_{2}$ of one-qubit unistochastic maps, which forms a non-convex subset of the tetrahedron of bistochastic maps spanned by three Pauli matrices and the identity map. In section IV the ensemble of random two-qubit quantum gates is described. It is based on circular unitary ensemble of special unitary matrices of size four. The probability distribution of purity (nonlocality) for this ensemble is computed. Furthermore, we derive the probability that a generic gate belongs to the class of perfect entanglers, so it can transform a product state into a maximally entangled Bell-like state. Unitary gates acting on $N \times N$ systems are investigated in Section V. For completeness, some basic properties of the operator Schmidt decomposition and related algebra of reshuffling of a matrix are reviewed in Appendix A.

\section{UNISTOCHASTIC MAPS}

Any unitary matrix $U$ of size $N^{2}$ describes a unitary gate acting on the bi-partite system. Alternatively it may be used to define a unistochastic map [25] acting on a single system of size $N$ according to eq. (1). In other words, the principal system is coupled to the ancilla of the same size, $M=N$, prepared initially in the maximally mixed state. Unless the gate $U$ is local so that $U=$ $U_{a} \otimes U_{b}$, the partial trace leads to a non-unitary evolution of the density matrix $\rho$.

Any such discrete map can be written in the Kraus form 34 ]

$$
\rho^{\prime}=\Phi(\rho)=\sum_{i=1}^{k} A_{i} \rho A_{i}^{\dagger} .
$$

To preserve the trace, $\operatorname{Tr} \rho^{\prime}=\operatorname{Tr} \rho=1$, the Kraus operators need to satisfy the completeness relation

$$
\sum_{i=1}^{k} A_{i}^{\dagger} A_{i}=\mathbb{1} .
$$

A trace preserving map $\Phi$ written in the form (2) is called stochastic. It can be represented by the dynamical matrix $D_{\Phi}=N(\Phi \otimes \mathbb{I})\left|\psi_{+}\right\rangle\left\langle\psi_{+}\right|$, also called Choi matrix. 
To perform the partial trace over the environment in the definition (10) let us apply the operator Schmidt decomposition of $U$ recalled in (A3). making use the notation introduced in Appendix the map $\Phi$ can be rewritten as

$$
\begin{gathered}
\rho^{\prime}=\Phi_{U} \rho=\operatorname{Tr}_{\mathrm{env}}\left[U\left(\rho \otimes \frac{1}{N} \mathbb{1}_{N}\right) U^{\dagger}\right] \\
=\operatorname{Tr}_{\mathrm{env}}\left[\sum_{i=1}^{N^{2}} \sum_{j=1}^{N^{2}} \sqrt{\Lambda_{i} \Lambda_{j}}\left(B_{i}^{\prime} \rho B_{j}^{\prime \dagger}\right) \otimes\left(\frac{1}{N} B_{i}^{\prime \prime} B_{j}^{\prime \prime \dagger}\right)\right] \\
=\frac{1}{N} \sum_{i=1}^{N^{2}} \Lambda_{i} B_{i}^{\prime} \rho B_{i}^{\prime \dagger} .
\end{gathered}
$$

The standard Kraus form is obtained by rescaling the operators, $A_{i}=\sqrt{\Lambda_{i} / N} B_{i}^{\prime}$, where $B_{i}^{\prime}$, which arise by reshaping the eigenvectors of $\left(U^{R}\right)^{\dagger} U^{R}$ according to (A4). Reshaped Kraus operators are eigenvectors of the hermitian dynamical matrix $D_{n \nu}$ of size $N^{2}$ which determines [35] the map

$$
\rho^{\prime}=\Phi(\rho) \quad \text { so } \quad \rho_{m \mu}^{\prime}=D_{\mu \nu}^{m n} \rho_{n \nu} .
$$

Taking into account an appropriate normalization we obtain the dynamical matrix corresponding to the unistochastic map

$$
D_{\Phi_{U}}=\frac{1}{N}\left(U^{R}\right)^{\dagger} U^{R}
$$

Hence the rescaled Schmidt coefficients $\Lambda_{i} / N$ of any unitary matrix $U$ treated as an element of the composite space of matrices of size $N^{2}$ provide the spectrum of the dynamical matrix $D$ representing the action of the unistochastic map $\Psi_{U}$.

The matrix $D$ is normalized according to $\operatorname{Tr} D=$ $\sum_{i=1}^{N^{2}} \frac{1}{N} \Lambda_{i}=N$. Therefore the entanglement entropy (A8) characterizing the non-local properties of $U$ is equal to the entropy of an operation $\Phi_{U}$ [25]. For instance, the entropy vanishes for any local $U$ which induces an unitary $\Phi_{U}$ and is equal to $2 \ln N$ for the Fourier matrix A10, corresponding the the maximally depolarizing channel, $\Psi_{F}(\rho)=\mathbb{1} / N$.

The environmental representation (1) may be generalized by allowing a larger size of the environment. Their physical motivation is simple: not knowing anything about the environment (apart from its dimensionality), one assumes that it is initially in the maximally mixed state. In particular, one can define generalized, $K$-unistochastic maps determined by a unitary matrix $U\left(N^{K+1}\right)$, in which the environment of size $N^{K}$ is initially in the state $\mathbb{1}_{N^{K}} / N^{K}$. By definition, a 1unistochastic map is unistochastic.

A map $\Phi$ is called bistochastic if it preserves the trace and keeps the maximally mixed state invariant, $\Psi(\mathbb{1} / N)=\mathbb{1} / N$. The definition (1) implies that both these conditions are satisfied, so any unistochastic map is bistochastic. In the following sections of this work we demonstrate that the converse is not true.
In the simplest case of one qubit maps any bistochastic map is called a Pauli channel, since it can be brought by means of unitary rotations into the form 36]

$$
\rho \rightarrow \rho^{\prime}=\sum_{i=0}^{3} \lambda_{i} \sigma_{i} \rho \sigma_{i} \quad \text { with } \quad \sum_{i=0}^{3} \lambda_{i}=1 .
$$

Here $\sigma_{i}$ denote Pauli matrices while $\sigma_{0}=\mathbb{1}$. The Pauli matrices satisfy $\sigma_{j}=-i \exp \left(i \pi \sigma_{j} / 2\right)$, so the extreme points of the set of the bistochastic maps represent rotations of the Bloch ball around the corresponding axis by the angle $\pi$.

Describing density matrices through their Bloch vectors, $\rho=\frac{1}{2} \mathbb{1}+\vec{\tau} \cdot \vec{\sigma}$, we can write any bistochastic map $\rho^{\prime}=\Phi \rho$ in the form

$$
\vec{\tau}^{\prime}=t \vec{\tau}=O_{1} \eta O_{2}^{\mathrm{T}} \vec{\tau} .
$$

Here $t$ denotes a real matrix of size 3 which we bring to a diagonal form by orthogonal transformations $O_{1}$ and $O_{2}$. As we permit only unitary rotations of the qubit represented by orthogonal matrices from $S O(3)$, (reflections are not allowed), some elements of the diagonal matrix $\eta$ may be negative. The elements of the diagonal matrix $\eta$ are called the damping vector $\vec{\eta}=\left(\eta_{1}, \eta_{2}, \eta_{3}\right)$, because the transformation (8) takes the Bloch ball to an ellipsoid with three axis given by $\vec{\eta}$.

Writing down the superoperator $\Phi$ of a Pauli channel and reshuffling this matrix according to (A6) we obtain the dynamical matrix $D$. For any bistochastic maps the dynamical matrix $D$ splits into two blocks and its eigenvalues are

$$
\begin{aligned}
d_{1,4} & =\frac{1}{2} \Lambda_{1,4}=\frac{1}{2}\left[1+\eta_{z} \pm\left(\eta_{x}+\eta_{y}\right)\right], \\
d_{2,3}=\frac{1}{2} \Lambda_{2,3} & =\frac{1}{2}\left[1-\eta_{z} \pm\left(\eta_{x}-\eta_{y}\right)\right] .
\end{aligned}
$$

Due to Choi theorem the map $\Phi$ is completely positive if the dynamical matrix $D$ is positive definite. This is the case if all eigenvalues $d_{i}=\Lambda_{i} / 2$ are not negative, which is true for the damping vector $\vec{\eta}$ satisfying the FujiwaraAlgoet conditions 37]

$$
\left(1 \pm \eta_{3}\right)^{2} \geq\left(\eta_{1} \pm \eta_{2}\right)^{2}
$$

These four inequalities assure that the corresponding positive map $\Phi_{\vec{\eta}}$ is CP. They define a regular tetrahedron whose extreme points are $\vec{\eta}=(1,1,1),(1,-1,-1)$, $(-1,1,-1),(-1,-1,1)$. The first point represents the identity operation while the three others correspond to unitary rotations by one of three Pauli matrices. As shown in section ЩВ there are bistochastic maps given by $\vec{\eta}$ inside the tetrahedron, for which the representation (11) does not exist, so they are not unistochastic.

\section{TWO QUBIT UNITARY GATES}

Treating unitary matrices as quantum gates we need not to care about an overall phase, since physical states 
differing by such a phase are identified. Discussing gates acting on a bipartite system we may thus fix this phase and restrict our attention to matrices pertaining to the special unitary group $S U\left(N^{2}\right)$.

In this section we are going to analyze the simplest case of two-qubit unitary gates. Hence we set $N=2$ and study matrices of the group $S U(4)$. A choice of two subspaces distinguishes a subgroup $S U(2) \otimes S U(2)$. From the physical point of view it corresponds to selection of two distinct subsystems.

\section{A. Canonical form}

Consider unitary matrices $U$ and $V$ of size $N^{2} \times N^{2}$ which act in the composite Hilbert space $\mathcal{H}_{N} \otimes \mathcal{H}_{N}$. Two such matrices are locally equivalent, written $U \sim_{\text {loc }} V$, if there exist local operations, $W_{A} \otimes W_{B}$ and $W_{C} \otimes W_{D}$, such that $V=\left(W_{A} \otimes W_{B}\right) U\left(W_{C} \otimes W_{D}\right)$.

It is known, [2, 18, 21], that any unitary matrix $U$ of size 4 is locally equivalent to some matrix of the following canonical form,

$$
V=\exp \left(i \sum_{k=1}^{3} \alpha_{k} \sigma_{k} \otimes \sigma_{k}\right):=\exp \left(i H_{\text {int }}\right)
$$

where $\sigma_{k}$ stand for the Pauli matrices, and $\alpha_{k}$, are real for $k=1,2,3$. Here $H_{\text {int }}$ denotes a Hermitian operator of order four, called the interaction Hamiltonian of the two-qubit gate.

The two-qubit gate $V$ is periodic in each parameter $\alpha_{k}$ with period $\pi / 2$. Thus the space of parameters characterizing the gate has a structure of a 3-torus $T^{3}$. However, the coefficients $\alpha_{k}$ are not determined unambiguously. Due to the symmetries of the problem out of a cube spanned by three components of $\vec{\alpha}$ one can distinguish a smaller subset, called Weyl chamber, such that the correspondence between its points and the local orbits is one-to-one [21]. For instance, it is possible to bring locally the Hamiltonian $H_{\text {int }}$ into a form in which [4]

$$
\begin{gathered}
\frac{\pi}{4} \geq \alpha_{1} \geq \alpha_{2} \geq \alpha_{3} \geq 0 \quad \text { or } \\
\frac{\pi}{2} \geq \alpha_{1}>\frac{\pi}{4}, \quad \frac{\pi}{2}-\alpha_{1} \geq \alpha_{2} \geq \alpha_{3} \geq 0 .
\end{gathered}
$$

These restrictions imply that the Weyl chamber has a structure of a tetrahedron, which forms a 1/24-th part of the cube - see Fig 1a.

The three-component vector $\vec{\alpha}$, called interaction content of the gate, characterize the purely non-local interaction Hamiltonian $H_{\text {int }}$. By construction two unitary gates are locally equivalent if and only if they are characterized by the same interaction content.

For completeness we present a direct algorithm of finding the information content $\vec{\alpha}$ of any two-qubit unitary gate $U$ based on 3,21$]$. It relays on the fact from the group theory, $S U(2) \times S U(2) \sim S O(4)$, which implies

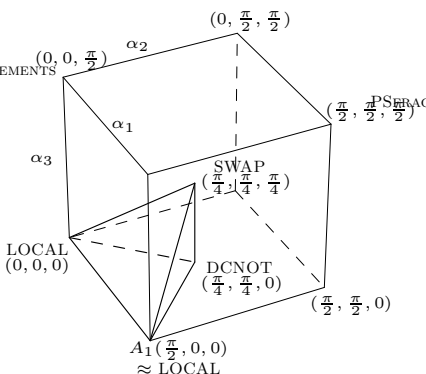

(a)

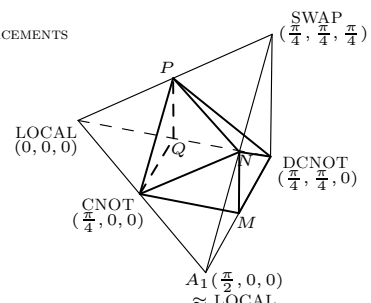

(b)
FIG. 1: a) Any orbit of locally equivalent two-qubit unitary gates intersects the Weyl chamber forming a tetrahedron in the cube of vectors $\vec{\alpha}$ representing the interaction content. b) The set of perfect entanglers forms a polytope inside the Weyl chamber with corners at points $P, Q, N, M$, CNOT and DCNOT [4]. Note that point $P$ represents $\sqrt{\text { SWAP. }}$

that any local operation from $S U(2) \times S U(2)$ forms in the so-called magic basis an orthogonal matrix $O \in S O(4)$ 38. Therefore any unitary $U$ written in this basis can be brought to the canonical form (11) by an orthogonal rotation,

$$
V=O^{T} U O
$$

The algorithm works for any $U \in U(4)$ and consist of five steps:

i) Find $U^{\prime}=U e^{-i \chi / 4}$ with $\chi$ equal to the phase of the $\operatorname{det} U$, such that $U^{\prime} \in S U(4)$;

ii) Write it down in the magic basis, i.e. find $W=$ $M U^{\prime} M^{\dagger}$, where $M=\frac{1}{\sqrt{2}}\left(\begin{array}{cccc}0 & -i & -i & 0 \\ 1 & 0 & 0 & 1 \\ -i & 0 & 0 & i \\ 0 & 1 & -1 & 0\end{array}\right)$, contains (row-wise) four Bell states forming the magic basis $\left\{-i\left|\psi^{+}\right\rangle,\left|\phi^{+}\right\rangle,-i\left|\phi^{-}\right\rangle,\left|\psi^{-}\right\rangle\right\}$;

iii) Compute $W W^{T}$ and find its spectrum, which we will write in the form $\left\{e^{-2 i \delta_{1}}, e^{-2 i \delta_{2}}, e^{-2 i \delta_{3}}, e^{-2 i \delta_{4}}\right\}$;

iv) Find vector $\vec{\delta}$ by dividing eigenphases of $W W^{T}$ by minus two. Pay attention to the total phase: By construction $\sum_{i} \delta_{i}$ should be equal to zero, so if this is not the case replace $\delta_{\max }$ by $\delta_{\max }-\pi$ or $\delta_{\min }$ by $\delta_{\min }+\pi$, which corresponds to another choice of the signs in $\sqrt{e^{2 i \delta}}$.

v) Change variables to obtain the information content $\vec{\alpha}$,

$$
\left\{\begin{array}{l}
\alpha_{1}=\left(\delta_{1}+\delta_{2}-\delta_{3}-\delta_{4}\right) / 4=\left(\delta_{1}+\delta_{2}\right) / 2 \\
\alpha_{2}=\left(\delta_{1}-\delta_{2}+\delta_{3}-\delta_{4}\right) / 4=\left(\delta_{1}+\delta_{3}\right) / 2 \\
\alpha_{3}=\left(-\delta_{1}+\delta_{2}+\delta_{3}-\delta_{4}\right) / 4=\left(\delta_{2}+\delta_{3}\right) / 2
\end{array}\right.
$$

Alternatively, the vector $\delta$ can be defined as the spectrum of the Hamiltonian $H_{i n t}$ entering the canonical form (11). The Hamiltonian $H_{i n t}$ is traceless and diagonal in the magic basis. Its four eigenvalues $\delta_{i}$ depend on the information content $\vec{\alpha}$ in a linear way. An inverse of (15) 
gives

$$
\left\{\begin{array}{l}
\delta_{1}=\alpha_{1}+\alpha_{2}-\alpha_{3} \\
\delta_{2}=\alpha_{1}-\alpha_{2}+\alpha_{3} \\
\delta_{3}=-\alpha_{1}+\alpha_{2}+\alpha_{3} \\
\delta_{4}=-\alpha_{1}-\alpha_{2}-\alpha_{3}
\end{array}\right.
$$

so one may easily switch between both representations.

Note that the existence of the canonical form (111) for $N=2$ is due to the fact that the group $S U(2)$ is homomorphic to $S O(3)$. However, for higher dimensions $S U(N)$ is homomorphic to a measure zero, $\left(N^{2}-1\right)-$ dimensional proper subset of the $\left(N^{2}-1\right)\left(N^{2}-2\right) / 2$ dimensional group $S O\left(N^{2}-1\right)$, and no direct analogue of such a canonical form is known.

\section{B. Schmidt coefficients}

By definition (A3) the Schmidt vector $\vec{\lambda}=\vec{\Lambda} / 4$ is invariant with respect to local unitary operations. To find its a relation with the information content $\vec{\alpha}$ of an arbitrary unitary matrix of size $N=4$ it suffices to take $U$ in its canonical form (11), and find singular values of the reshuffled matrix $U^{R}$. Their squares appear in the spectral decomposition of a positive, Hermitian matrix

$U_{\text {can }}^{R}\left(U_{\text {can }}^{R}\right)^{\dagger}=\Lambda_{1} \mathbb{I} \otimes \mathbb{I}+\Lambda_{2} \sigma_{1} \otimes \sigma_{1}+\Lambda_{3} \sigma_{2} \otimes \sigma_{2}+\Lambda_{4} \sigma_{3} \otimes \sigma_{3}$

Simple algebra gives the connection between local invariants:

$\left\{\begin{array}{c}\Lambda_{1}=\left(1+\cos 2 \alpha_{2} \cos 2 \alpha_{3}+\cos 2 \alpha_{1} \cos 2 \alpha_{3}+\cos 2 \alpha_{1} \cos 2 \alpha_{2}\right) \\ \Lambda_{2}=\left(1+\cos 2 \alpha_{2} \cos 2 \alpha_{3}-\cos 2 \alpha_{1} \cos 2 \alpha_{3}-\cos 2 \alpha_{1} \cos 2 \alpha_{2}\right) \\ \Lambda_{3}=\left(1-\cos 2 \alpha_{2} \cos 2 \alpha_{3}+\cos 2 \alpha_{1} \cos 2 \alpha_{3}-\cos 2 \alpha_{1} \cos 2 \alpha_{2}\right) \\ \Lambda_{4}=\left(1-\cos 2 \alpha_{2} \cos 2 \alpha_{3}-\cos 2 \alpha_{1} \cos 2 \alpha_{3}+\cos 2 \alpha_{1} \cos 2 \alpha_{2}\right)\end{array}\right.$

Introducing new variables

$$
\left\{\begin{array}{l}
\eta_{1}:=\cos 2 \alpha_{2} \cos 2 \alpha_{3} \\
\eta_{2}:=\cos 2 \alpha_{1} \cos 2 \alpha_{3} \\
\eta_{3}:=\cos 2 \alpha_{1} \cos 2 \alpha_{2}
\end{array}\right.
$$

we realize that relations (18) are equivalent to (9). Thus the coordinates $\vec{\eta}$ used above represent the damping vector which enters Eq. (8) and characterizes the corresponding unistochastic map $\Phi_{U}$. Note that the vector $\vec{\Lambda}$ of Schmidt coefficients of $U$, determines the eigenvalues $d_{i}=\Lambda_{i} / N$ of the dynamical matrix $D_{U}$ associated with $\Phi_{U}$, while $\lambda_{i}=\Lambda_{i} / N^{2}$ describe the weights in the Pauli channel (7).

To see which damping vectors $\vec{\eta}$ correspond to unistochastic maps we have to describe the image of the Weyl chamber (13) with respect to transformation (19). We may invert this relation

$$
\left\{\begin{array}{l}
\alpha_{1}=\frac{1}{2} \arccos \frac{\eta_{2} \eta_{3}}{\eta_{3}} \\
\alpha_{2}=\frac{1}{2} \arccos \frac{\eta_{1} \eta_{3}}{\eta_{2}} \\
\alpha_{3}=\frac{1}{2} \arccos \frac{\eta_{1} \eta_{2}}{\eta_{3}}
\end{array}\right.
$$

if the absolute value of arguments of arc cosine are smaller then unity. This leads to the following restrictions for the set $\mathcal{U}$ of damping vectors corresponding to unistochastic maps

$$
\left\{\begin{array}{l}
\eta_{1} \eta_{2} \leq \eta_{3} \\
\eta_{2} \eta_{3} \leq \eta_{1} \\
\eta_{3} \eta_{1} \leq \eta_{2}
\end{array}\right.
$$

A bistochastic one-qubit map $\Phi_{\vec{\eta}}$ is unistochastic, if the vector $\vec{\eta}$ satisfies the set of three conditions (21), so relation (20) gives the interaction content $\vec{\alpha}$ and the explicit form (11) of the unitary matrix defining the map.

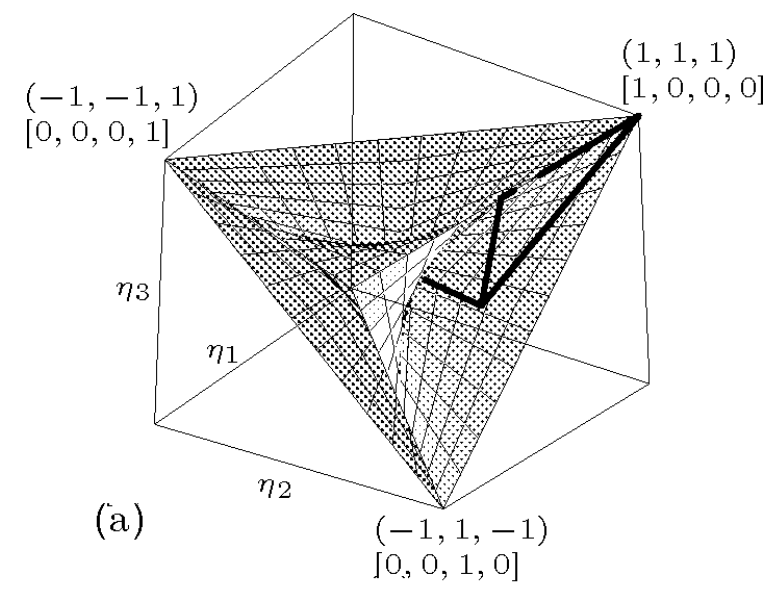

(b)

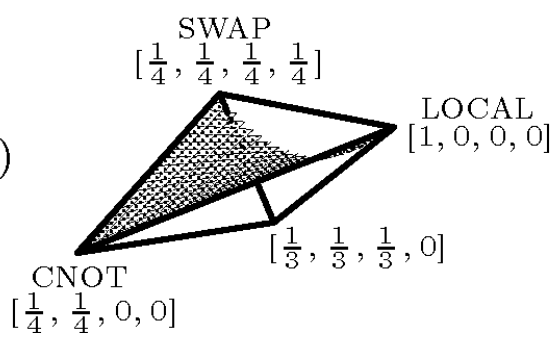

FIG. 2: a) The set $\mathcal{U}_{2}$ of one-qubit unistochastic maps forms a proper subset of the tetrahedron of bistochastic maps. b) The part of the Weyl chamber of non-equivalent unistochastic maps.

The set $\mathcal{U}_{2}$ of one-qubit unistochastic maps forms a proper subset of the tetrahedron of bistochastic maps bounded by three parabolic hyperboloids $\eta_{1}=\eta_{2} \eta_{3}$, $\eta_{2}=\eta_{1} \eta_{3}$, and $\eta_{3}=\eta_{1} \eta_{2}$. Note that the set $\mathcal{U}_{2}$ is not convex: it contains all four corners and six edges of the tetrahedron, but its center, the point $\vec{\eta}=0$, belongs to the boundary of $\mathcal{U}_{2}$ - see Fig 2 . Thus we may model $\mathcal{U}_{2}$ by pressing symmetrically four faces of a tetrahedron with rigid edges in such a way that they all touch in the center - see Figs. 2 and 3.

Let us now try to invert relations (19) and (20) to obtain the vector $\vec{\alpha}$ as function of the components of the 


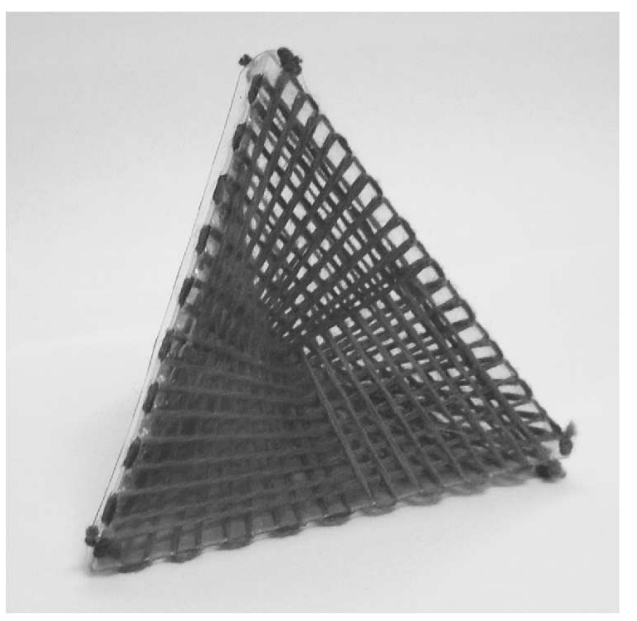

FIG. 3: A model of the set $\mathcal{U}_{2}$ of one-qubit unistochastic maps which forms a non-convex subset of the tetrahedron of bistochastic maps. The model relays on the fact that the hyperboloids (21) can be ruled by straight lines.

Schmidt vector,

$\left\{\begin{array}{lll}\alpha_{1}=\frac{1}{2} \arccos \sqrt{w_{1}} & \text { where } & w_{1}=\frac{\left(\Lambda_{1}+\Lambda_{4}-2\right)\left(\Lambda_{1}+\Lambda_{3}-2\right)}{2\left(\Lambda_{1}+\Lambda_{2}-2\right)}, \\ \alpha_{2}=\frac{1}{2} \arccos \sqrt{w_{2}} & \text { where } & w_{2}=\frac{\left(\Lambda_{1}+\Lambda_{4}-2\right)\left(\Lambda_{1}+\Lambda_{2}-2\right)}{2\left(\Lambda_{1}+\Lambda_{3}-2\right)} \\ \alpha_{3}=\frac{1}{2} \arccos \sqrt{w_{3}} & \text { where } & w_{3}=\frac{\left(\Lambda_{1}+\Lambda_{2}-2\right)\left(\Lambda_{1}+\Lambda_{3}-2\right)}{2\left(\Lambda_{1}+\Lambda_{4}-2\right)}\end{array}\right.$

The choice of the sign of the square root determines the sign of $\alpha_{3}$. Constraints for unistochasticity (21) imply that $\left|w_{i}\right| \leq 1$ for $i=1,2,3$ so the function arccos is well-defined. Alternatively, these inequalities provide constraints $\vec{\lambda}$ has to satisfy, to represent a Schmidt vector of a $N=4$ unitary matrix. The choice (13) of the domain containing $\vec{\alpha}$ implies that the elements of the Schmidt vector are ordered non-increasingly, $\Lambda_{1} \geq \Lambda_{2} \geq \Lambda_{3} \geq \Lambda_{4}$.

A local unitary operation $(\vec{\alpha}=0)$ is of rank one, while a generic two-qubit unitary gate has Schmidt rank 4. As noted by Dür and Cirac [22] and Nielsen and al. [9],

The data presented in the table may be easily obtained for an arbitrary unitary matrix $U$ : the spectrum of $U U^{R}$ gives the Schmidt vector $\Lambda$, the phases $U U^{T}$ (normalized in such a way that their sum vanishes) provide vector $\vec{\delta}$, while the information content $\vec{\alpha}$ follows from relations (15).

Note that the matrices $U_{\mathrm{DCNOT}}$ and $U_{\mathrm{SWAP}}$ are invariant with respect to reshuffling, so the reshuffled matrix is unitary and all four its singular values are equal to unity. Reshuffled Fourier matrix remains unitary, so it has the same singular values. Hence the Fourier matrix, the gates DCNOT and SWAP are characterized by the same, maximally mixed Schmidt vector $\vec{\Lambda}_{*}=(1,1,1,1)$, but they there are no unitary matrices of Schmidt rank 3. Indeed, by setting $\Lambda_{4}$ to zero, equations (22) impose $\Lambda_{3}=0$ as well. Interestingly, for two-qutrit composite systems there exist unitary gates of size 9 with Schmidt rank equal to $r$ for each $r=1, \ldots, 9$ [39].

Observe that expressions (22) are ill defined if all components are equal, $\vec{\Lambda}_{*}=(1,1,1,1)$. Hence one may expect that this degenerated Schmidt vector of the maximal entropy, $S=2 \ln 2$, corresponds to different, non-locally equivalent gates. This fact, discussed in [22], may be demonstrated with explicit examples. Let us define the following two-qubit unitary gates

$$
\begin{gathered}
U_{\mathrm{CNOT}}=\left[\begin{array}{llll}
1 & 0 & 0 & 0 \\
0 & 1 & 0 & 0 \\
0 & 0 & 0 & 1 \\
0 & 0 & 1 & 0
\end{array}\right], \quad U_{\mathrm{DCNOT}}=\left[\begin{array}{llll}
1 & 0 & 0 & 0 \\
0 & 0 & 0 & 1 \\
0 & 1 & 0 & 0 \\
0 & 0 & 1 & 0
\end{array}\right], \\
U_{\text {SWAP }}=\left[\begin{array}{llll}
1 & 0 & 0 & 0 \\
0 & 0 & 1 & 0 \\
0 & 1 & 0 & 0 \\
0 & 0 & 0 & 1
\end{array}\right]
\end{gathered}
$$

Their names are related to applications in the theory of quantum information [1]. The gate CNOT performs the control not operation: $U_{\mathrm{CNOT}}|a, b\rangle=|a, a \oplus b\rangle$ where $a$ is the control bit, $b$ is the target bit and the sum is understood modulo two. Defining a symmetric CNOT operation with the role of bits reversed, $U_{\mathrm{CNOT}^{\prime}}|a, b\rangle=$ $|a \oplus b, a\rangle$, one defines the double CNOT gate by the composition $U_{\mathrm{DCNOT}}=U_{\mathrm{CNOT}} U_{\mathrm{CNOT}^{\prime}}$. The SWAP gate may be written as a product of three CNOT gates, $U_{\mathrm{SWAP}}=U_{\mathrm{CNOT}^{\prime}} U_{\mathrm{CNOT}} U_{\mathrm{CNOT}^{\prime}}$, while its action on any two qubits, $U_{\mathrm{SWAP}}|a, b\rangle=|b, a\rangle$ explains its name. Characterization of non-local properties of these exemplary two-qubit gates is provided in Table 1, complementary to the data listed in [40]. For comparison we include also the so-called $B$-gate which interpolates between CNOT and DCNOT and is optimal to simulate an arbitrary twoqubit gate [4].

carry different information content, and thus are not locally equivalent 22]. Making use of (22) we infer that any gate with the information content $\vec{\alpha}=(\pi / 4, \pi / 4, x)$ with an arbitrary $x$ is characterized by the same maximally mixed Schmidt vector $\Lambda_{*}$.

For comparison we have provided the data for matrices representing $\sqrt{\mathrm{CNOT}}$ and $\sqrt{\mathrm{SWAP}}$, which may be obtained by replacing the fragments of (23) containing the NOT gate $U_{\mathrm{NOT}}=\left[\begin{array}{ll}0 & 1 \\ 1 & 0\end{array}\right]$ by $U_{\sqrt{\mathrm{NOT}}}:=$ $\frac{1}{2}\left[\begin{array}{cc}1+i & i-1 \\ 1-i & 1+i\end{array}\right]$. In these cases taking the square root of a gate corresponds to dividing its interaction content by 
TABLE I: Nonlocal properties of two-qubit unitary quantum gates; $s_{ \pm}=2 \pm \sqrt{2}, t=1 / \sqrt{2}$; perfect entanglers: No (N), Yes inside the set (Y), Yes - at the boundary (B).

\begin{tabular}{lcccccc}
\hline \hline Gates & $\begin{array}{c}\text { information } \\
\text { content } \vec{\alpha}\end{array}$ & $\begin{array}{c}\text { Hamiltonian } \\
\text { eigenvalues } \vec{\delta}\end{array}$ & $\begin{array}{c}\text { Schmidt } \\
\text { vector } \vec{\Lambda}\end{array}$ & $\begin{array}{c}\text { Schmidt damping } \\
\text { rank }\end{array}$ & $\begin{array}{c}\text { perfect } \\
\text { vector } \vec{\eta}\end{array}$ & entangler \\
\hline local gate & $(0,0,0)$ & $(0,0,0,0)$ & $(4,0,0,0)$ & 1 & $(1,1,1)$ & $\mathrm{N}$ \\
$\sqrt{\text { CNOT }}$ & $\frac{\pi}{8}(1,0,0)$ & $\frac{\pi}{8}(1,1,-1,-1)$ & $\left(s_{+}, s_{-}, 0,0\right)$ & 2 & $(1, t, t)$ & $\mathrm{N}$ \\
CNOT & $\frac{\pi}{8}(2,0,0)$ & $\frac{\pi}{8}(2,2,-2,-2)$ & $(2,2,0,0)$ & 2 & $(1,0,0)$ & $\mathrm{B}$ \\
B-gate & $\frac{\pi}{8}(2,1,0)$ & $\frac{\pi}{8}(3,1,-1,-3)$ & $\frac{1}{2}(3,3,1,1)$ & 4 & $\frac{1}{2}(1,0,0)$ & $\mathrm{Y}$ \\
DCNOT & $\frac{\pi}{8}(2,2,0)$ & $\frac{\pi}{8}(4,0,0,-4)$ & $(1,1,1,1)$ & 4 & $(0,0,0)$ & $\mathrm{B}$ \\
$\sqrt{\text { SWAP }}$ & $\frac{\pi}{8}(1,1,1)$ & $\frac{\pi}{8}(1,1,1,-3)$ & $\frac{1}{2}(5,1,1,1)$ & 4 & $\frac{1}{2}(1,1,1)$ & $\mathrm{B}$ \\
SWAP & $\frac{\pi}{8}(2,2,2)$ & $\frac{\pi}{8}(2,2,2,-6)$ & $(1,1,1,1)$ & 4 & $(0,0,0)$ & $\mathrm{N}$ \\
Fourier & $\frac{\pi}{8}(2,2,-1)$ & $\frac{\pi}{8}(5,-1,-1,-3)$ & $(1,1,1,1)$ & 4 & $(0,0,0)$ & $\mathrm{N}$ \\
\hline \hline
\end{tabular}

two, $\vec{\alpha}\left(U_{\sqrt{\text { SWAP }}}\right)=\frac{1}{2} \vec{\alpha}\left(U_{\text {SWAP }}\right)$. More generally, among several possibilities of taking a $k$-th root of an unitary matrix $U$ described by $\vec{\alpha}$ one can select an unitary matrix $U^{1 / k}$ such that its interaction content equals $\vec{\alpha} / k$.

Let us mention here that the entangling power of integer roots of the SWAP gate were investigated in [42]. Another possibility to characterize nonlocal properties of unitary gates by so-called Frobenius fidelity was recently investigated in [17].

\section{SCOE(4) - ENSEMBLE OF TWO-QUBIT UNITARY GATES}

In this section we study random two-qubit unitary gates described by unitary matrices of size $N=4$. An ensemble of unitary matrices generated according to the Haar measure is called 43] circular unitary ensemble (CUE).

We aim to derive the probability distributions of local invariants of unitary matrices of size 4 . Since quantum states are defined up to a global phase, we may restrict our attention to the set of special unitary matrices $S U(4)$ with $\operatorname{det} U=1$. An ensemble containing special unitary matrices with $\operatorname{det} U=1$ induced by the Haar measure will be called SCUE.

To find the distribution $P(\vec{\alpha})$ we use magic basis and the representation given by Eq. (14). The Haar measure on CUE(4) induces in set of symmetric unitary matrices $Y=U U^{T}$ the measure

$$
d_{H} Y=d_{H} O^{T} Y O
$$

invariant with respect to orthogonal similarity $Y \rightarrow$ $O^{T} Y O$. A set endowed with such invariant volume is called circular orthogonal ensemble (COE), so the ensemble of symmetric unitary matrices with determinant equal to unity will be called SCOE.

It is known 43] that random symmetric unitary matrices of $\mathrm{COE}$ are characterized by the following joint distribution of eigenphases $\Theta_{i}$

$$
\begin{array}{r}
P\left(\Theta_{1}, \Theta_{2}, \Theta_{3}, \Theta_{4}\right)=\mathcal{N} \delta\left(\Theta_{1}+\Theta_{2}+\Theta_{3}+\Theta_{4}\right) \\
\prod_{1 \leq m<n \leq 4}\left|e^{i \Theta_{n}}-e^{i \Theta_{m}}\right|
\end{array}
$$

where $\mathcal{N}$ is the normalization constant.

Integrating over $\Theta_{4}$ we obtain

$$
\begin{array}{r}
P\left(\Theta_{1}, \Theta_{2}, \Theta_{3}\right)=\mathcal{N}^{\prime}\left|e^{i\left(-\Theta_{1}-\Theta_{2}-\Theta_{3}\right)}-e^{i \Theta_{3}}\right| \\
\times\left|e^{i\left(-\Theta_{1}-\Theta_{2}-\Theta_{3}\right)}-e^{i \Theta_{2}}\right|\left|e^{i\left(-\Theta_{1}-\Theta_{2}-\Theta_{3}\right)}-e^{i \Theta_{1}}\right| \\
\times\left|e^{i \Theta_{3}}-e^{i \Theta_{2}}\right|\left|e^{i \Theta_{3}}-e^{i \Theta_{1}}\right|\left|e^{i \Theta_{2}}-e^{i \Theta_{1}}\right| .
\end{array}
$$

The angles $\pi / 2 \geq \Theta_{i} \geq \pi / 2, i:=1,2,3,4$ are the eigenvalues of $Y=U U^{T}$, hence $\Theta_{i}=2 \delta_{i}[3]$. Thus

$$
\begin{aligned}
\alpha_{1} & =\frac{1}{4}\left(\delta_{1}+\delta_{2}-\delta_{3}-\delta_{4}\right)=\frac{1}{4}\left(\Theta_{1}+\Theta_{2}\right) \\
\alpha_{2} & =\frac{1}{4}\left(\delta_{1}-\delta_{2}+\delta_{3}-\delta_{4}\right)=\frac{1}{4}\left(\Theta_{1}+\Theta_{3}\right) \\
\alpha_{3} & =\frac{1}{4}\left(-\delta_{1}+\delta_{2}+\delta_{3}-\delta_{4}\right)=\frac{1}{4}\left(\Theta_{2}+\Theta_{3}\right),
\end{aligned}
$$

i.e.

$$
\begin{aligned}
& \Theta_{1}=2\left(\alpha_{1}+\alpha_{2}-\alpha_{3}\right) \\
& \Theta_{2}=2\left(\alpha_{1}-\alpha_{2}+\alpha_{3}\right) \\
& \Theta_{3}=2\left(-\alpha_{1}+\alpha_{2}+\alpha_{3}\right) .
\end{aligned}
$$

In this way we obtain one of the main results of this paper: the joint probability distribution for the interaction content vector $\vec{\alpha}$, induced by the Haar measure on $S U(4)$

$$
\begin{aligned}
P\left(\alpha_{1}, \alpha_{2}, \alpha_{3}\right) & =\mathcal{N}^{\prime \prime}\left|\sin \left(2\left(\alpha_{1}+\alpha_{2}\right)\right)\right|\left|\sin \left(2\left(\alpha_{1}+\alpha_{3}\right)\right)\right| \\
& \times\left|\sin \left(2\left(\alpha_{2}+\alpha_{3}\right)\right)\right|\left|\sin \left(2\left(\alpha_{1}-\alpha_{2}\right)\right)\right| \\
& \times\left|\sin \left(2\left(\alpha_{1}-\alpha_{3}\right)\right)\right|\left|\sin \left(2\left(\alpha_{2}-\alpha_{3}\right)\right)\right| .(33)
\end{aligned}
$$

The resulting normalization constant $\mathcal{N}^{\prime \prime}$ can be easily calculated to be $2 / \pi$ by integrating over the cube $-\pi \leq$ $\Theta_{i} \leq \pi$, i.e. $-\pi / 2 \leq c_{i} \leq \pi / 2, i=1,2,3$. 
Making use of (19) one can change variables and get the probability distribution of the damping vector $\vec{\eta}$,

$$
P\left(\eta_{1}, \eta_{2}, \eta_{3}\right)=\frac{\left|\frac{\eta_{1} \eta_{2}}{\eta_{3}}-\frac{\eta_{3} \eta_{1}}{\eta_{2}}\right|\left|\frac{\eta_{3} \eta_{1}}{\eta_{2}}-\frac{\eta_{2} \eta_{3}}{\eta_{1}}\right|\left|\frac{\eta_{2} \eta_{3}}{\eta_{1}}-\frac{\eta_{1} \eta_{2}}{\eta_{3}}\right|}{\left|1-\frac{\eta_{2} \eta_{3}}{\eta_{1}}\right|\left|1-\frac{\eta_{1} \eta_{2}}{\eta_{3}}\right|\left|1-\frac{\eta_{1} \eta_{2}}{\eta_{3}}\right|}
$$

defined for vectors $\vec{\eta}$ satisfying constraints (21). This expression shows that the density is concentrated in vicinity of the boundary of the set $\mathcal{U}_{2}$ inscribed inside the tetrahedron of one-qubit bistochastic maps. Alternatively, one may easily get the analytical expression $P(\lambda)$ for the density inside the Schmidt simplex induced by the Haar measure on $S U(4)$, but we found it more convenient to work in the $\vec{\eta}$ representation and to use (34).

This distribution may be applied to compute average values of various measures of nonlocality of random twoqubit unitary gate. Consider, for instance the purity $r$ (locality) related to the linear entropy of the Schmidt vector

$$
r:=\lambda_{1}^{2}+\lambda_{2}^{2}+\lambda_{3}^{2}+\lambda_{4}^{2}=\frac{1}{4}\left(1+\eta_{1}^{2}+\eta_{2}^{2}+\eta_{3}^{2}\right) .
$$

The average purity of a unitary matrix distributed according to the Haar measure on $U\left(N^{2}\right)$ was computed by Zanardi [7]

$$
\langle r\rangle_{N}=\frac{2}{N^{2}+1},
$$

so in the case of two-qubit gates this average reads $\langle r\rangle_{2}=$ $2 / 5$.

Making use of the joint distribution (34) we can go a step further and get the entire probability distribution $P(r)$ as a triple integral. Two integrals are easy to perform analytically, but the last integral had to be computed numerically. Results shown as a line in Fig. IV are compared with the Monte Carlo calculations in which $10^{5}$ random unitary matrices of size 4 where generated and the distribution of their locality collected into the histogram denoted by black dots. Results obtained with both methods agree well and show that a typical random unitary gate has low purity, so it is strongly non-local. There exits a large class of quantum gates with the linear entropy $r \sim 3 / 8$, close to the value for which the analyzed probability distribution $P(r)$ achieves its maximal value. All these gates represent a 'generic' behavior in the entire set of two-qubit unitary quantum gates, but it is hardly possible to distinguish out of them a single gate with some special properties.

\section{THE SUBSET OF PERFECT ENTANGLERS OF TWO-QUBIT UNITARY GATES}

Among all two-qubit gates represented by unitary matrices of size 4, one may distinguish so-called perfect entanglers, which can produce from a product state a maximally entangled, Bell-like state. A gate $U$ belongs to this

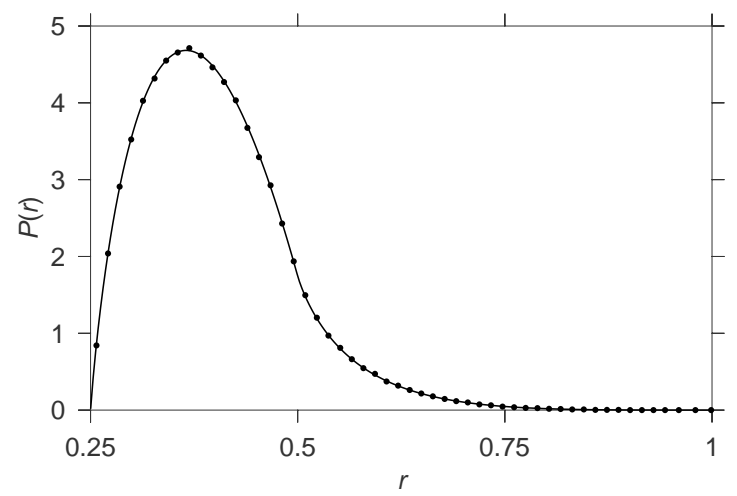

FIG. 4: Probability distribution of purity $r$ of random unitary matrices distributed according to the Haar measure on $S U(4)$. Solid line represents numerical integration of distribution (34), while black dots show the histogram obtained by a Monte-Carlo approach.

class if its numerical range (for a normal matrix equal to the convex hull of the spectrum) includes the eigenvalue $z=0$ [18]. In terms of the parameters $\alpha_{1}, \alpha_{2}, \alpha_{3}$ the condition reads [4]

$$
\frac{\pi}{4}-\left|\frac{\pi}{4}-\alpha_{1}\right| \geq \frac{\pi}{8}-\left|\frac{\pi}{8}-\alpha_{2}\right| \geq \alpha_{3} \geq 0,
$$

In the latter work the authors have shown that the relative volume of the set of perfect entanglers is equal to $1 / 2$. However, they used the uniform measure in the 3-dimensional space of the vectors representing the information content (or rather its relevant part, called Weyl chamber), which does not corresponds the natural Haar measure on the set of unitary matrices.

In this section we redo the calculations using the measure $P\left(\alpha_{1}, \alpha_{2}, \alpha_{3}\right)$ induced by the Haar measure on $U(N)$ and given by Eq. (33).

As explained in Section III the set of nonequivalent gates is parametrized by $\alpha_{1}, \alpha_{2}, \alpha_{3}$ restricted by inequalities (13), i.e. belonging to the tetrahedron $T_{0}$ with vertices $O=(0,0,0), A=(\pi / 2,0,0), U_{\mathrm{DCNOT}}=$ $(\pi / 4, \pi / 4,0)$, and $U_{\mathrm{SWAP}}=(\pi / 4, \pi / 4, \pi / 4)$. Hence its volume with respect to the measure (33) equals:

$$
\begin{aligned}
V_{w} & =\int_{0}^{\pi / 4} d \alpha_{1} \int_{0}^{\alpha_{1}} d \alpha_{2} \int_{0}^{\alpha_{2}} d \alpha_{3} P\left(\alpha_{1}, \alpha_{2}, \alpha_{3}\right) \\
& +\int_{\pi / 4}^{\pi / 2} d \alpha_{1} \int_{0}^{\pi / 2-\alpha_{1}} d \alpha_{2} \int_{0}^{\alpha_{2}} d \alpha_{3} P\left(\alpha_{1}, \alpha_{2}, \alpha_{3}\right)=\frac{\mathcal{N}}{24} .
\end{aligned}
$$

The set of the perfect entanglers (PE) given by (37) is the convex polyhedron with vertices: $L=(\pi / 4,0,0)$, $M=(3 \pi / 8, \pi / 8,0), U_{\mathrm{DCNOT}}, Q=(\pi / 8, \pi / 8,0)$, $N=(3 \pi / 8, \pi / 8, \pi / 8)$, and $P=(\pi / 8, \pi / 8, \pi / 8)$, and a straightforward calculation of its volume with respect to the measure (33) gives:

$$
V_{p e}=\frac{\mathcal{N}}{9 \pi} .
$$


Hence the relative volume of the perfect entanglers reads

$$
\frac{V_{p e}}{V_{w}}=\frac{8}{3 \pi} \approx 0.85
$$

and gives the probability with which a typical two-qubit gate is a perfect entangler. This value, much larger than one half, is consistent with the earlier observations, that a generic unitary gate is highly non-local.

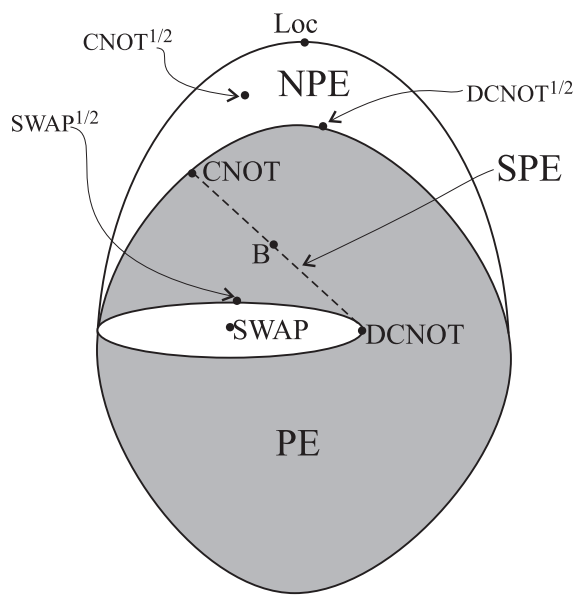

FIG. 5: A sketch of the set of two-qubit unitary quantum gates: the set of perfect entanglers (PE) occupies approximately $85 \%$ of the entire volume and contains the set SPE of measure zero. The complementary set of non perfect entanglers (NPE) consists of two (connected) parts which include local gates (Loc) and SWAP operation, respectively.

In the set of two-qubit gates one distinguishes also the set of special perfect entanglers (SPE), which can maximally entangle a set of four product states which form an orthogonal basis 44. Special perfect entanglers form a one dimensional set of gates interpolating between CNOT and DCNOT, so the natural measure of this set is equal to zero. The interpolating gate $B$, introduced in [41], is located inside of the set of PE and belongs as well to SPE - see Fig. 5. which shows a sketch of the set of two-qubit unitary gates.

\section{VI. $\quad N \times N$ SYSTEMS \& TWO QUNITS UNITARY GATES}

Studying non-local properties of a unitary gate $U$ of an arbitrary size $N \times N$ of higher dimensions we have to relay on its Schmidt decomposition, since for $N \geq 3$ no direct analogue of the canonical form (11) exists. The Schmidt vector, equal to the squared singular values of the reshuffled matrix $U^{R}$, in several cases may be found analytically.

\section{A. Schmidt vectors for exemplary gates}

Analyzing, for instance,the general case of the swap operator $U_{\text {SWAP }}$ acting now in the space of two quNits, SWAP $|a, b\rangle=|b, a\rangle$, we see that $U_{\mathrm{SWAP}}^{R}=U_{\mathrm{SWAP}}$. Hence the reshuffled matrix is unitary, so all $N$ of its singular values are equal to unity and the entanglement entropy is maximal, $S\left(U_{\text {SWAP }}\right)=2 \ln N$. Selecting one element out of $N$ in each block of the first $N$ rows of $U$ it is straightforward to write down the other $N !-1$ permutation matrices, which are invariant with respect to the reshuffling transformation, so their entanglement entropy is maximal.

The same property is also characteristic of the Fourier matrix of order $N^{2}$ with entries $F_{k l}=\frac{1}{N} \exp \left(2 \pi k l / N^{2}\right)$. Also in this case it is enough to see that the reshuffled matrix $F^{R}$ remains unitary, which implies $S(F)=2 \ln N$. This result was earlier established in [9, 39]. The fact that the entropy of the SWAP gate and of the Fourier matrix are maximal, does not imply that both gates are locally equivalent. Up till now the general question which unitary gates of size $K N>4$ are locally equivalent remains open.

Among other two-quNits gates let us mention two possible generalizations of the CNOT (XOR) gate [45]

$$
U_{+}|i, j\rangle \equiv|i, i \oplus j\rangle \quad \text { and } \quad U_{-}|i, j\rangle \equiv|i, i \ominus j\rangle,
$$

where $i, j=0,1, \cdots, N-1$ and both operations are taken modulo $N$.

Since addition and subtraction of bits modulo two are equivalent, both gates coincide for $N=2$ with the standard CNOT gate defined in (23). The gate $U_{-}$may be called a 'controlled rotation', because the target bit $j$ gets rotated depending on the value of the control bit $i$ and $\left(U_{-}\right)^{d}=\mathbb{1}$. Inasmuch as the gate $U_{+}$is symmetric and forms an involution, $\left(U_{+}\right)^{2}=\mathbb{1}$, it was called the generalized XOR gate by Alber et al. [46], who demonstrated that $U_{+}$is capable of performing various tasks of quantum information processing and proposed a physical realization of this gate based on non-linear optical elements.

Both gates are permutation matrices with the block diagonal structure. In both cases all blocks of size $N$ reshaped into vectors of length $N$ become mutually orthogonal, so the matrix $U_{ \pm}^{R}\left(U_{ \pm}^{R}\right)^{\dagger}$ is diagonal and consist of exactly $N$ non-zero elements, each of them equal to $N$. This fact implies that the entanglement entropy of both gates is equal and reads

$$
S\left(U_{+}\right)=S\left(U_{-}\right)=\ln N .
$$

\section{B. Random gates and average values}

The Haar measure on the set of matrices of a composite size $M N$ determines a natural measure on the set of unitary quantum gates. A gate taken randomly with respect to this measure will be non-local with probability 
equal to one, since the set of local gates of the tensor product structure, $U_{1}(N) \otimes U_{2}(M)$, forms only a zero measure in $U(N M)$. On the other hand, one may ask to what extend a generic unitary gate is non-local. Formulating this question more precisely, we will investigate mean values of entropies of entanglement averaged with respect to the Haar measure.

The average purity (36) implies that the average linear entropy $E=1-\sum_{i=1}^{N^{2}} \lambda_{i}^{2}$ reads $\langle E\rangle_{N}=\left(N^{2}-1\right) /\left(N^{2}+1\right)$ [7]. Interestingly, this average is equal to the mean purity of squared components of a random complex vector of size $N^{2}$, distributed according to the natural, unitarily invariant measure on the space of pure states. However, we will demonstrate that both distributions are different. In particular, our numerical results show that the average entropy of entanglement (A8), behaves like

$$
\langle S\rangle_{U} \sim 2 \ln N-\frac{1}{2}
$$

while the mean entropy of a random vector of the size $N^{2}$ reads

$$
\langle S\rangle_{\phi}=\Psi\left(N^{2}+1\right)-\Psi(2)=\sum_{k=2}^{N^{2}} \frac{1}{k} \approx 2 \ln N+1-\gamma
$$

In this formula, derived in [47], $\Psi(x)$ denotes the digamma function while $\gamma \approx 0.5772$ is the Euler constant.

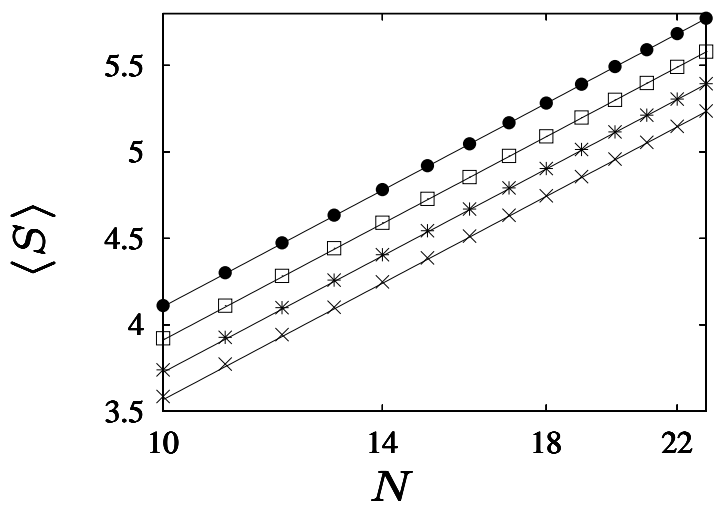

FIG. 6: Mean Rényi entropies of entanglement averaged over unitary matrices acting on symmetric $N \times N$ systems: $(\bullet)$ represents the mean Shannon entropy $\langle S\rangle$, while $(\square),(*)$ and $\times$ denote average Renyi entropy $\left\langle S_{q}\right\rangle$ for $q$ equal to 2,4 and 8 respectively. Dimension $N$ is plotted in the log scale, and solid lines represent the asymptotic behavior.

To characterize statistical distribution of the distribution of Schmidt vectors of random unitary gates we computed the average moments $\left\langle\sum_{i} \lambda_{i}^{q}\right\rangle$ and the average Rényi entropy (A9). Our results show that the average entropy for the $N \times N$ unitary gates behaves as $\left\langle S_{q}\right\rangle \approx 2 \ln N-c_{q}-$ see Fig. 6] Since the dimension $N$ is plotted in the logarithmic scale, relations (44) correspond to straight lines in the graph. The values of the constants $c_{q}$ agree with predictions $c_{1}=1 / 2, c_{2}=\ln 2 \approx 0.69$, $c_{4}=(\ln 14) / 3 \approx 0.88$ derived in [48] for the average Renyi entropy of mixed quantum states of size $N^{2}$ obtained by partial trace of random pure states of an extended system. This fact shows that a random positive matrix $U^{R}\left(U^{R}\right)^{\dagger}$ has properties of a Wishart random matrix: for a large matrix size the unitarity of $U$ hardly influences statistical property of the corresponding reshuffled matrix $U^{R}$, which can be treated as a typical non-Hermitian matrix from the Ginibre ensemble [4], as their statistical properties coincide asymptotically.

A generic quantum pure state $|\psi\rangle$ of a bipartite $N \times N$ system is typically highly entangled, so the corresponding reduced density matrix, $\rho=\operatorname{Tr}_{N}|\psi\rangle\langle\psi|$, is highly mixed and its von Neumann entropy is close to the maximal value, $\ln N$. This known fact [48, 50] is directly related to properties of random unitary operation: a generic unitary gate $U$ acting on $N \times N$ system is highly nonlocal and the corresponding unistochastic operation $\Psi_{U}$ is strongly depolarizing.

Above observations hold also for a general case of $k$ unistochastic channels or quantum gates acting on $N \times$ $M$ systems. The Haar measure on the unitary group $U(N M)$ induces a certain measure on the simplex $\Delta_{N^{2}}$ containing all possible Schmidt vectors of size $N^{2}$. The larger size $M$ of the auxiliary subsystem, the larger is the average Shannon entropy of the Schmidt vector $\Lambda^{\prime}$, equal to the average entangling power of the quantum gate. In the limit $M \rightarrow \infty$ the average entanglement entropy tends to the maximal value, $2 \ln N$.

\section{CONCLUDING REMARKS}

In this paper we have analyzed the ensemble of unitary quantum gates distributed uniformly with respect to the Haar measure on the unitary group. As the overall phase of the matrix does not influence its action on a quantum state, we restricted our attention to unitary matrices with determinant equal to unity and considered matrices from special circular unitary ensemble (SCUE).

A generic unitary gate $U$, acting on a bipartite $N \times N$ system, was shown to be strongly non-local. For instance, its entanglement entropy $S(U)$, (also called Schmidt strength [9]), behaves as $2 \ln N-1 / 2$, which is also characteristic of random density matrices of size $N^{2}$, distributed according to the flat measure [48].

Any bi-partite quantum gate $U$, represented by a unitary matrix of size $N^{2}$, determines a unistochastic operation $\Phi_{U}$, in which the initial state of size $N$ is coupled by $U$ with the environment of the same size, prepared in a maximally mixed state [25]. The entanglement entropy of a gate $S(U)$, equal to the entropy $S\left(\Phi_{U}\right)$ of the corresponding unistochastic channel (4), is given by the Shannon entropy of the eigenvalues of $U^{R}\left(U^{R}\right)^{\dagger} / N^{2}$. The reshuffled matrix $U^{R}$ is non-Hermitian and for a Haar random unitary $U$, its statistical properties are shown to coincide with predictions of the Ginibre ensemble. Thus 
the spectral density of a normalized, Wishart-like matrix $U^{R}\left(U^{R}\right)^{\dagger} / N^{2}$, is asymptotically described by the Marchenko-Pastur distribution.

Analyzing in particular the set of two-qubit unitary gates we derived an explicit formula for the joint probability density (33) for the interaction content vector $\vec{\alpha}$ characterizing a quantum gate, and the distribution (34) for the damping vector $\vec{\eta}$. Having these results at hand we were in position to analyze the subset of bi-partite quantum gates consisting of perfect entanglers - unitary gates capable to transform a separable state into a maximally entangled Bell-like state. Using the known conditions for a two qubit gate to be a perfect entangler [4, 18], we found that according to the natural Haar measure on the unitary group the set of perfect entanglers occupies approximately $85 \%$ of the entire volume of the space of unitary matrices of order four. This observation gives a concrete argument supporting the claim that local gates are rather exceptional, while a generic unitary gate is strongly non local.

Although any two locally equivalent unitary matrices possess the same set of the Schmidt coefficients and generate the same unistochastic map, the reverse is not true. For instance, as shown in Table 1, the following two-qubit gates: SWAP, DCNOT and the Fourier matrix are characterized by the uniform vector $\Lambda$ of the Schmidt coefficients so their entanglement entropy is equal to $2 \ln 2$. Although these unitary matrices are characterized by different information content $\alpha$, so they are not locally equivalent, these gates generate the same unistochastic map: the maximally depolarizing channel.

Any unistochastic operation, determined by a unitary matrix acting on an extended space, is by construction bistochastic. However, not every bistochastic operation is unistochastic and can be obtained by a partial trace over the environment of the same size, initially prepared in the maximally mixed state. In the case of one qubit maps, there are no unistochastic maps of rank three, represented by a point belonging to the face of the tetrahedron of bistochastic maps, spanned by three Pauli matrices and identity - see Fig. 3. This is consistent with the known fact that there is no two-qubit unitary gates of Schmidt rank three [9, 22]. For instance the symmetric Pauli channel,

$$
\rho \rightarrow \rho^{\prime}=\frac{1}{3} \sum_{i=1}^{3} \sigma_{i} \rho \sigma_{i}
$$

at the center of the face of the tetrahedron of bistochastic maps is located as far from the set $\mathcal{U}_{2}$ of one-qubit unistochastic operations as possible.

It is a pleasure to thank I. Bengtsson and H.- J. Sommers for fruitful discussions and to N. Johnston for pointing out relevant references. Support by the grant number N N202-090-239 of Polish Ministry of Science and Higher Education and by SFB/Transregio-12 program financed by Deutsche Sonderforschungs Gemeinschaft is gratefully acknowledged.

\section{Appendix A: Schmidt decomposition of a unitary operator}

\section{Matrix algebra: reshaping}

Consider a rectangular matrix $A_{i j}, j=1, \ldots, N$ and $i=1, \ldots, M$. Equivalently, one may put its elements row after row into a vector $\vec{a}_{k}$ of size $M N$,

$$
A_{i j}=\vec{a}_{(i-1) N+j}
$$

For instance, a unitary matrix $U$ of size $M \times M$ will be thus transformed into a vector $\vec{u}$ with $M^{2}$ components.

Let $\mathcal{H}_{N}$ denote an $N$ dimensional complex Hilbert space, and $\mathcal{H}_{H S}$ the corresponding $N^{2}$ dimensional Hilbert-Schmidt space of all linear operators acting on $\mathcal{H}_{N}$. It is equipped with a scalar product $A \cdot B=$ $\langle A \mid B\rangle:=\operatorname{tr} A^{\dagger} B$, where $A$ and $B$ are arbitrary complex matrices of size $N \times N$.

Let $V$ denote an auxiliary unitary matrix of size $N^{2}$. Unitarity of $V$ implies that its $N^{2}$ columns $\vec{b}_{m}=V_{i m}$ (or rows $\vec{b}_{m}=V_{m i}$ ), $m, i=1, \ldots N^{2}$ reshaped into square $N \times N$ matrices $B_{m}$ as in (A1), form an orthonormal basis in $\mathcal{H}_{H S}$, since $\left\langle B_{m} \mid B_{n}\right\rangle:=\operatorname{Tr} B_{m}^{\dagger} B_{n}=\delta_{m n}$. Note that the matrices $B_{m}$ need not to be unitary.

Consequently, the tensor products $\left(B_{m} \otimes B_{n}\right), m, n=$ $1, \ldots, N^{2}$, span an orthonormal basis in the composite Hilbert-Schmidt space $\mathcal{H}_{H S} \otimes \mathcal{H}_{H S}$ of size $N^{4}$ in which acts the original $N^{2} \times N^{2}$ unitary matrix $V$.

\section{Operator Schmidt decomposition}

Take a given unitary matrix $U$ of size $N^{2} \times N^{2}$ we wish to investigate. It belongs to the composite HilbertSchmidt space $\mathcal{H}_{H S} \otimes \mathcal{H}_{H S}$ and will be occasionally denoted as $|U\rangle$. Let us write down its representation in the basis defined above,

$$
|U\rangle=\sum_{m=1}^{N^{2}} \sum_{n=1}^{N^{2}} C_{m n}\left|B_{m}\right\rangle \otimes\left|B_{n}\right\rangle
$$

where $C_{m n}=\operatorname{tr}\left(\left(B_{m} \otimes B_{n}\right)^{\dagger} U\right)$. The complex matrix $C$ of size $N^{2} \times N^{2}$ needs not to be Hermitian nor normal. The Schmidt decomposition of $|U\rangle$ reads

$$
|U\rangle=\sum_{k=1}^{N^{2}} \sqrt{\Lambda_{k}}\left|B_{k}^{\prime}\right\rangle \otimes\left|B_{k}^{\prime \prime}\right\rangle,
$$

where $\sqrt{\Lambda_{k}}$ are the singular values of $C$, (square roots of eigenvalues of the positive matrix $C^{\dagger} C$ ) and the basis is transformed by a local unitary transformation $W_{a} \otimes W_{b}$. Thus $\left|B^{\prime}\right\rangle=W_{a}|B\rangle$, and $\left|B^{\prime \prime}\right\rangle=W_{b}|B\rangle$ where $W_{a}$ and $W_{b}$ are matrices composed of eigenvectors of $C^{\dagger} C$ and $C C^{\dagger}$, respectively. In a typical case of a non-degenerate spectrum of $C C^{\dagger}$, the Schmidt decomposition is unique 
up to two unitary diagonal matrices, up to which the matrices of eigenvectors $W_{a}$ and $W_{b}$ are determined.

Note that the matrix $C$ depends on the initial basis, $\left\{B_{m} \otimes B_{n}\right\}_{m, n=1}^{N}$, in which the analyzed matrix $U$ is represented, while the Schmidt coefficients $\Lambda_{k}$ are basis independent. Thus, for convenience we may analyze the special case in which the basis in $\mathcal{H}_{H S}$ is generated by the identity matrix, of size $N^{2} \times N^{2}$. Then each of the $N^{2}$ basis matrices $B_{n}$ of size $N \times N$ has only one non vanishing element which equals unity. Let's denote $B_{k}=B^{m \mu}=|m\rangle\langle\mu|$, where $k=N(m-1)+\mu$. In this case the matrix of the coefficients $C$ has a particularly simple form, $C_{n \nu} \underset{n \nu}{ }=\operatorname{Tr}\left(B^{m \mu} \otimes B^{n \nu}\right) U=U_{\mu \nu}$.

\section{Matrix algebra: reshuffling}

This particular reordering of a matrix, called reshuffling [25], will be denoted as $U^{R}:=C$. In general the notion of reshuffling is well defined if a matrix $X$ acts on a composite Hilbert space, $\mathcal{H}_{M} \otimes \mathcal{H}_{N}$. The symbol $U^{R}$ has a unique meaning if a concrete decomposition of the total dimension, $L=M N$, is specified. Similar reorderings of matrices were considered by Hill et al. [51, 52. while investigating CP maps and also in [53 57] to analyze separability of mixed quantum states.

The Schmidt coefficients of $U$ are equal to the squared singular values of the reshuffled matrix, $U^{R}$. Therefore the operator Schmidt decomposition (A3) of an arbitrary matrix $X$ may be summarized by

$$
\left\{\begin{array}{ccc}
\left\{\sqrt{\Lambda_{k}}\right\}_{k=1}^{N^{2}} & =\text { singular values of } X^{R} \\
\left|B_{k}^{\prime}\right\rangle & =\text { reshaped eigenvectors of }\left(X^{R}\right)^{\dagger} X^{R} \\
\left|B_{k}^{\prime \prime}\right\rangle & =\text { reshaped eigenvectors of } X^{R}\left(X^{R}\right)^{\dagger}
\end{array} .\right.
$$

Note that the singular values of the reshuffled matrix, $\mathrm{SV}\left(X^{R}\right)$, are equal to square roots of eigenvalues of a positive matrix $\left(X^{R}\right)^{\dagger} X^{R}$. The initial basis is transformed by a local unitary transformation $W_{a} \otimes W_{b}$, where $W_{a}$ and $W_{b}$ are matrices of eigenvectors of matrices $\left(X^{R}\right)^{\dagger} X^{R}$ and $X^{R}\left(X^{R}\right)^{\dagger}$, respectively. If and only if the rank $K$ of $X^{R}\left(X^{R}\right)^{\dagger}$ is equal to one, the operator can be factorized into a product form, $X=X_{1} \otimes X_{2}$, where $X_{1}=\operatorname{Tr}_{2} X$ and $X_{2}=\operatorname{Tr}_{1} X$.

To get a better feeling of the reshuffling transformation observe that reshaping each row of the initial matrix $X$ of length $N^{2}$ according to (A1) into a submatrix of size $N$ and placing it according to the lexicographical order block after block produces the reshuffled matrix $X^{R}$. Let us illustrate this procedure for the simplest case $N=2$, in which any row of the matrix $X$ is reshaped into a $2 \times 2$ matrix

$$
C_{k j}=X_{k j}^{R}:=\left[\begin{array}{cc|lc}
\mathbf{X}_{11} & \mathbf{X}_{\mathbf{1 2}} & X_{21} & X_{22} \\
X_{13} & X_{14} & \mathbf{X}_{\mathbf{2 3}} & \mathbf{X}_{\mathbf{2 4}} \\
\hline \mathbf{X}_{\mathbf{3 1}} & \mathbf{X}_{\mathbf{3 2}} & X_{41} & X_{42} \\
X_{33} & X_{34} & \mathbf{X}_{\mathbf{4 3}} & \mathbf{X}_{\mathbf{4 4}}
\end{array}\right] .
$$

The operation of reshuffling could be defined in an alternative way. Instead of reshaping the vectors of $X$ into square matrices of size $N$ one can reshape columns of $X$, which leads to another reshuffled matrix $X^{R^{\prime}}$. In the four indices notation introduced above (Roman indices running from 1 to $N$ correspond to the first subsystem, Greek indices to the second one), both operations of reshuffling take the form

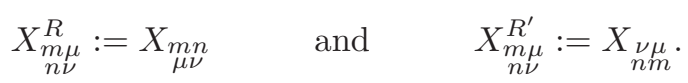

However, both reshuffled matrices are equivalent up to a certain permutation of rows and columns and transposition, so the singular values of $X^{R^{\prime}}$ and $X^{R}$ are equal. It is easy to see that $\left(X^{R}\right)^{R}=X$. In general, $N^{3}$ elements of $X$ do not change their position during the operation of reshuffling (these typeset boldface in (A5)); the other $N^{4}-N^{3}$ elements do. The space of complex matrices with the reshuffling symmetry is thus $2 N^{4}-2\left(N^{4}-N^{3}\right)=2 N^{3}$ dimensional. Note that if $X$ is Hermitian the reshuffled matrix $X^{R}$ needs not to be Hermitian.

\section{Entanglement entropy}

The Hilbert-Schmidt norm of any unitary matrix is $\|U\|=\sqrt{\langle U \mid U\rangle}=\sqrt{N}$. Computing the norm of the right hand side of (A3) we obtain

$$
\sum_{k=1}^{N^{2}} \Lambda_{k}=N^{2}
$$

Thus the normalized vector $\vec{\lambda}$ of the squared singular values, $\lambda_{k}:=\Lambda_{k} / N^{2}$, lives in the $\left(N^{2}-1\right)$ dimensional simplex and may be interpreted as a probability vector. Iff there exists only one non-zero singular value, $\lambda_{1}=1$, then the unitary matrix has a product form, $U=U_{a} \otimes U_{b}$. In such a case $U$ is called a local gate and both operators obtained by partial tracing, $U_{a}=\operatorname{Tr}_{b} U$ and $U_{b}=\operatorname{Tr}_{a} U$ are unitary.

In general, the vector of the Schmidt coefficients of an unitary matrix $U$ acting on a composite $N \times N$ system conveys information concerning the non-local properties of $U$. To characterize quantitatively the distribution of $\vec{\lambda}$ one uses the Shannon entropy,

$$
S(U):=S(\vec{\lambda})=-\sum_{k=1}^{N^{2}} \lambda_{k} \ln \left(\lambda_{k}\right)
$$

called in this context entanglement entropy of $U$ [7], (or Schmidt strength [9]), and the generalized, Rényi entropies

$$
S_{q}(U):=S_{q}(\vec{\lambda})=-\frac{1}{1-q} \ln \left[\sum_{k=1}^{N^{2}}\left(\lambda_{k}\right)^{q}\right],
$$


which tend to $S$ in the limit $q \rightarrow 1$. The entropy $S_{0}$, sometimes called Hartley entropy, is equal to $\ln L$, where $L$ denotes the number of positive coefficients $\lambda_{i}$, and is called Schmidt rank (or Schmidt number).

The second order Renyi entropy $S_{2}$ is closely related to the linear entropy $E(U)=1-\exp \left(-S_{2}\right)$ used by Zanardi in [7]. The quantity $r=\sum_{k=1}^{N^{2}}\left(\lambda_{k}\right)^{2}$ is called purity while analyzing the vector of eigenvalues of an arbitrary density matrix $\rho$ : the larger coefficient $r$, the more pure state. The maximal value, $r=1$ is attained if and only if the state $\rho$ is pure. In the present analysis of the unitary matrices, we shall stick to this name, although in this context $r$ could be termed locality: the gate $U$ is local if and only if $r=1$. Another quantity called inverse participation ratio is usefull: $R=1 / r=\exp \left(S_{2}\right)$ varies from unity (local gates) to $N^{2}$ for the Fourier unitary matrices of size $N^{2}$ defined by

$$
F_{k l}^{\left(N^{2}\right)}:=\frac{1}{N} \exp \left(i 2 \pi k l / N^{2}\right)
$$

To demonstrate this fact it is sufficient to notice that the reshuffled matrix $F^{R}$ remains unitary, so all its singular values are equal to unity, hence the Schmidt vector contains $N^{2}$ equal components and is maximally mixed. Some examples of unitary two-qubit gates and their Schmidt vector are collected in Table I.

\section{Local equivalence}

By virtue of the Schmidt decomposition if two gates are locally equivalent, their Schmidt coefficients (and thus the entanglement entropy) are equal. However, the opposite is not true: there exist unitary gates with the same set of Schmidt coefficients, which are not locally equivalent [22]. Hence equality of Schmidt vectors characterizing two unitary matrices of size $N^{2}$ is a necessary but not sufficient condition for their local equivalence. Sufficient conditions for local equivalence are known [2, 4, 21] only for $N=2$.
[1] M. A. Nielsen and I. L. Chuang, Quantum Computation and Quantum Information, Cambridge University Press, Cambridge, 2000.

[2] N. Khaneja, R. Brockett and S. J. Glaser, Time-optimal control in spin systems, Phys. Rev. A 63032308 (2001).

[3] K. Hammerer, G. Vidal and J.I. Cirac, Characterization of non-local gates, Phys. Rev. A 66, 062321 (2002).

[4] J. Zhang, J. Vala, K.B. Whaley and S. Sastry, A geometric theory of non-local two-qubit operations, Phys. Rev. A 67, 042313 (2003).

[5] X. Wang, B. C. Sanders, and D. W. Berry, Entangling power and operator entanglement in qudit systems, Phys. Rev. A 67, 042323 (2003).

[6] P. Zanardi, C. Zalka and L. Faoro, On the entangling power of quantum evolutions, Phys. Rev. A 62, 030301(R) (2000).

[7] P. Zanardi, Entanglement of quantum evolution, Phys. Rev. A 63, 040304(R), (2001).

[8] X. Wang and P. Zanardi, Quantum entanglement of unitary operators on bi-partite systems, Phys. Rev. A 66, 044303 (2002).

[9] M. A. Nielsen, C. M. Dawson, J. L. Dodd, A. Gilchrist, D. Mortimer, T. J. Osborne, M. J. Bremner, A. W. Harrow and A. Hines, Quantum dynamics as physical resource, Phys. Rev. A 67, 052301 (2003).

[10] A. J. Scott and C. M. Caves, Entangling power of the quantum baker's map, J. Phys. A 36, 9553 (2003).

[11] M. M. Wolf, J. Eisert, and M. B. Plenio, Entangling power of passive optical elements, Phys. Rev. Lett. 90, 047904 (2003).

[12] A. J. Scott, Multipartite entanglement, quantum-errorcorrecting codes, and entangling power of quantum evolutions, Phys. Rev. A 69, 052330 (2004).

[13] L. Clarisse, S. Ghosh, S. Severini, and A. Sudbery, Entangling Power of Permutations, Phys. Rev. A 72, 012314 (2005).
[14] J. Batle, M. Casas, A. Plastino, and A. R. Plastino, Entanglement Distribution and Entangling Power of Quantum Gates, Optics and Spectroscopy 99371 (2005).

[15] N. Linden, J. A. Smolin, and A. Winter, Entangling and disentangling power of unitary transformations are not equal, Phys. Rev. Lett. 103, 030501 (2009).

[16] A. Soeda and M. Murao, Delocalization power of global unitary operations on quantum information, New J. Phys. 12093013 (2010)

[17] H. Saberi, Ancilla-assisted sequential approximation of nonlocal unitary operations, Phys. Rev. A 84, 032323 (2011).

[18] Y. Makhlin, Nonlocal properties of two-qubit gates and mixed states, and the optimization of quantum computations, Quant. Inf. Proc. 1, 243 (2002).

[19] L. Yu, R. B. Griffiths, and S. M. Cohen, Efficient implementation of bipartite nonlocal unitary gates using prior entanglement and classical communication, Phys. Rev. A 81, 062315 (2010).

[20] S. M. Cohen, All maximally entangling unitary gates, Phys. Rev. A 84, 052308 (2011).

[21] B. Kraus and J. I. Cirac, Optimal creation of entanglement using a two-qubit gate, Phys. Rev. A 63062309 (2001).

[22] W. Dür and J. I. Cirac, Equivalence classes of non-local operations, Quant. Inf. Comp. 2, 240 (2002).

[23] W. Dür, G. Vidal and J. I. Cirac, Optimal conversion on non-local unitary operation, Phys. Rev. Lett. 89, 057901 (2002).

[24] M. Poźniak, K. Życzkowski, and M. Kuś, Composed ensembles of random unitary matrices, J. Phys. A 31, 1059 (1998).

[25] K. Życzkowski and I. Bengtsson, On duality between quantum states and quantum maps, Open Syst. Inf. Dyn. 11, 3-42 (2004). 
[26] K. Życzkowski, M. Kuś, W. Słomczyński and H.-J. Sommers, Random unistochastic matrices, J. Phys. A 36, 3425-3450 (2003).

[27] E. Knill and R. Laflamme, Power of one bit of quantum information, Phys. Rev. Lett. 81, 5672 (1998).

[28] D. Poulin, R. Blume-Kohout, R. Laflamme, and H. Olivier, Exponential speedup with a single bit of quantum information, Phys. Rev. Lett. 92, 177906 (2004).

[29] M. Horodecki, P. Horodecki and J. Oppenheim, Reversible transformations from pure to mixed states and the unique measure of information, Phys. Rev. A 67,062104 (2003).

[30] U. Haagerup and M. Mustat, Factorization and dilation problems for complete positive maps on von Neumann algebras, Commun. Math. Phys. 303 555-594 (2011).

[31] C. Anantharaman-Delaroche, On ergodic theorems for free group actions on noncommutative spaces, Probab. Theory Rel. Fields 135, 520-546 (2006).

[32] R. F. Werner, All teleportation and dense coding schemes, J. Phys. A 34, 7081 (2001).

[33] A. Wójcik, A. Grudka and R. Chhajlany, Generation of inequivalent generalized Bell bases, Quant. Inf. Proc. 2, 201 (2003).

[34] K. Kraus, General state changes in quantum theory, Ann. Phys. 64, 311 (1971).

[35] E. C. G. Sudarshan, P. M. Mathews, and J. Rau, Stochastic dynamics of quantum-mechanical systems, Phys. Rev. 121920 (1961).

[36] M. B. Ruskai, S. Szarek and E. Werner, An Analysis of completely-positive trace-preserving maps on $2 \times 2$ matrices, Linear Algebra Appl. 347, 159 (2002).

[37] A. Fujiwara and P. Algoet, One-to-one parametrization of quantum channels, Phys. Rev. A 59,3290 (1999).

[38] S. Hill and W. K. Wootters, Entanglement of a pair of quantum bits, Phys. Rev. Lett. 78, 5022 (1997).

[39] J. Tyson, Operator-Schmidt decomposition of the quantum Fourier transform on $\mathbb{C}^{N_{1}} \otimes \mathbb{C}^{N_{2}}$, J. Phys. A 36, 6485 (2003).

[40] S. Balakrishnan, R. Sankaranarayanan, Characterizing the geometrical edges of nonlocal two-qubit gates, Phys. Rev. A 79, 052339 (2009).

[41] J. Zhang, J. Vala, S. Sastry, and K. B. Whaley, Minimum construction of two-qubit quantum operation, Phys. Rev. Lett. 93, 020502 (2004).
[42] S. Balakrishnan and R. Sankaranarayanan, Entangling characterization of $\mathrm{SWAP}^{1 / m}$ and controlled unitary gates, Physical Review A 78, 052305 (2008).

[43] M. L. Mehta, Random Matrices, II ed., Academic Press, New York, 1991.

[44] A. T. Rezakhani, Characterization of two-qubit perfect entanglers, Phys. Rev. A 70, 052313 (2004).

[45] M. Horodecki and P. Horodecki, Reduction criterion of separability and limits for a class of distillation protocols, Phys. Rev. A 59, 4206 (1999).

[46] G. Alber, A. Delgado, N. Gisin, and I. Jex, Generalized quantum XOR-gate in arbitrary dimensional Hilbert space, J. Phys. A 34, 8821 (2001).

[47] K. R. W. Jones, Entropy of random quantum states, J. Phys. A23, L1247 (1990).

[48] K. Życzkowski and H.-J. Sommers, Induced measures in the space of mixed quantum states, J. Phys. A 34, 7111 (2001)

[49] P. J. Forrester, Log-gases and Random Matrices Princeton: Princeton University Press 2010

[50] P. Hayden, D. W. Leung and A. Winter, Aspects of generic entanglement, Commun. Math. Phys. 269, 95 (2006).

[51] C. J. Oxenrider and R. D. Hill, On the matrix reordering $\Gamma$ and $\Psi$, Linear Alg. Appl. 69, 205 (1985).

[52] D. A. Yopp and R. D. Hill, On completely copositive and decomposable linear transformations, Linear Alg. Appl. 312, 1 (2000).

[53] O. Rudolph, On the cross norm criterion for separability, J. Phys. A 36, 5825 (2003).

[54] O. Rudolph, Some Properties of the computable cross norm criterion for separability, Phys. Rev., A 67, 032312 (2003).

[55] K. Chen and L.-A. Wu, A matrix realignment method for recognizing entanglement, Quant. Inf. Comp. 3, 193 (2003).

[56] M. Horodecki, P. Horodecki, and R. Horodecki, Characterization of separable states: Linear contractions, and permutattion criteria, Open Systems Inform. Dynamics 13, 103 (2004).

[57] C. Lupo, P. Aniello, A. Scardiccio, Bipartite quantum states: on the realignment criterion and beyond, J. Phys. A 41, 415301 (2008). 